\title{
Prediction of response to bacillus Calmette-Guérin treatment in non-muscle invasive bladder cancer patients through interleukin-6 and interleukin-10 ratio
}

\author{
TOMMASO CAI ${ }^{1}$, GABRIELLA NESI $^{2}$, SANDRA MAZZOLI ${ }^{3}$, FRANCESCA MEACCI $^{3}$, GALLIANO TINACCI $^{4}$, \\ LORENZO GIUSEPPE LUCIANI ${ }^{1}$, VINCENZO FICARRA ${ }^{5}$, GIANNI MALOSSINI ${ }^{1}$ and RICCARDO BARTOLETTI $^{6}$ \\ ${ }^{1}$ Department of Urology, Santa Chiara Hospital, Trento; ${ }^{2}$ Division of Pathological Anatomy, \\ Department of Critical Care Medicine and Surgery, University of Florence, Florence; ${ }^{3} \mathrm{STD}_{\mathrm{s}}$ Center \\ and ${ }^{4}$ Department of Pathology, Santa Maria Annunziata Hospital, Florence; ${ }^{5}$ Department of Urology, \\ University of Padua, Padua; ${ }^{6}$ Department of Urology, University of Florence, Florence, Italy
}

Received September 13, 2011; Accepted December 27, 2011

DOI: $10.3892 /$ etm.2012.634

\begin{abstract}
This study aimed to evaluate whether the interleukin-6 (IL-6) and interleukin-10 (IL-10) ratio (IL-6/IL-10) can be used as a prognostic marker of recurrence following bacillus Calmette-Guérin (BCG) therapy in patients with high-risk non-muscle invasive bladder cancer (NMIBC). One hundred and twenty-one consecutive urological patients (72 affected by high-risk NMIBC and 49 controls) were selected for this prospective study. Urine samples for dipstick and interleukin analyses were collected from each subject before surgery. All patients underwent transurethral resection of bladder tumours (TUR-BT), followed by six weekly BCG instillations. IL-6 and IL-10 concentrations in urine were determined by solid phase ELISA Quantikine IL-6 and IL-10 Immunoassay. Patients with NMIBC were stratified in accordance with IL-6/IL-10: group A $\leq 0.09$ and group B $>0.10$. The main outcome measures were time to first recurrence and recurrence rate following BCG therapy. At enrolment, IL-6/IL-10 was not statistically different between patients and controls $(\mathrm{p}=0.763$, degrees of freedom $(\mathrm{df})=1, \mathrm{~F}$-test result $(\mathrm{F})=0.092)$. Of the 72 patients with NMIBC, 38 (52.7\%) had an IL-6/IL-10 of $\leq 0.09$ (group A), while $34(47.3 \%$ ) had an IL-6/IL-10 of $>0.10$ (group B). A significant difference between IL-6/IL-10 and status at follow-up was found $\left(\mathrm{p}=0.016, \mathrm{df}=1, \chi^{2}=5.800\right)$. The Kaplan-Meier curves demonstrated that group B patients had a significantly higher probability of being recurrence-free than group A patients $[\mathrm{p}=0.003$; recurrence rate $(\mathrm{RR})=3.1]$. At multivariate analysis, IL-6/IL-10 $(\mathrm{p}<0.003)$ and the number
\end{abstract}

Correspondence to: Dr Tommaso Cai, Department of Urology, Santa Chiara Hospital, Largo Medaglie d'Oro 9, 38123 Trento, Italy E-mail: ktommy@libero.it

Key words: interleukin-6, interleukin-10, bacillus Calmette-Guérin, bladder cancer, urinary bladder neoplasms, urothelial cancer, urinary cytology, recurrence of lesions $(\mathrm{p}<0.001)$ were identified as independent predictors of BCG response probability. In conclusion, this study highlights the feasible role of IL-6/IL-10 in predicting recurrence following BCG therapy in high-risk NMIBC.

\section{Introduction}

Intravesical bacillus Calmette-Guérin (BCG) immunotherapy is considered more effective than intravesical chemotherapy and is advocated as the first treatment choice in patients with carcinoma in situ or with pT1G3 (1). However, not all patients benefit from intravesical BCG (2). In fact, 30-50\% of patients undergoing intravesical $\mathrm{BCG}$ treatment have recurring tumours following therapy (3-5), with common side-effects that can be life threatening (6). Patients refractory to BCG therapy are subjected to salvage cystectomy as standard treatment (2), though may show a poor prognosis (7). Therefore, the patient failing BCG therapy is a challenge to the urologist and predictors of BCG response should be investigated (2). Although several authors have proposed molecular markers to predict the clinical outcome of patients with non-muscle invasive bladder cancer (NMIBC) following treatment with intravesical BCG (8-9), no marker has yet proven to be valid in everyday clinical practice (2). Zhang et al demonstrated the induction of cytokine production by BCG, particularly interleukin-6 (IL-6) (10). Recently, Luo et al showed the inhibitory role of interleukin-10 (IL-10) in BCG-induced macrophage cytotoxicity, suggesting that the blockage of IL-10 may potentially enhance the effect of BCG in the treatment of bladder cancer patients (11). Chen et al demonstrated that BCG increases IL-6 messenger RNA and protein in a timeand dose-dependent manner via an immediate early pathway (12). Furthermore, we highlighted the feasible role of IL-6 and IL-10 ratio (IL-6/IL-10) as a prognostic marker of recurrence in patients affected by NMIBC (9). Urinary interleukins could consequently be used as prognosticators to better assess the outcome of NMIBC patients following BCG therapy. The present study aimed to evaluate the role of IL-6/IL-10 in predicting patient response to BCG therapy. 


\section{Materials and methods}

Study design. To evaluate the prognostic role of urinary IL-6/IL-10, all consecutive patients who had undergone transurethral resection of bladder tumours (TUR-BT) at the same urological unit (Department of Urology, University of Florence, Florence, Italy) between January 2005 and May 2006 were selected for this prospective study. As controls, 49 subjects were enrolled from among patients attending the clinic in the same period for other non-malignant urological diseases. Since this study was designed to establish the role of IL-6/IL-10 as a predictor of the response to BCG and not as a diagnostic tool, patients with other urological malignant diseases were excluded. Outcome measures were time to first recurrence following treatment and recurrence rate. Disease progression was not included among the main outcomes due to the small number of patients who experienced stage or grade progression, though these data are reported in Results. Response to BCG was confirmed by negative urinary cytology and cystoscopy after 6 months' follow-up (13). According to Herr and Dalbagni, patients were defined as BCG-refractory if the recurrence occurred within 6 months of starting BCG treatment (14). Urine samples for the urine dipstick test and interleukin analyses were collected before TUR-BT and 6 subsequent weekly BCG instillations. Depending on laboratory results, all patients enrolled in the study were divided into 2 groups: group A with IL-6/IL-10 $\leq 0.09$ and group B with IL-6/IL-10 $>0.10$. The controls constituted group C. Follow-up data were compared and analysed. Patients with no further information available at follow-up were excluded from the study. The local research ethics committee approved the study and written informed consent was obtained from all patients.

Eligibility for the study. Inclusion criteria were the presence of cystoscopically demonstrated bladder tumours, normal pre-operative blood tests and compliance with instillation and follow-up schedules. We enrolled only patients affected by high-risk NMIBC, according to the European Association of Urology Guidelines criteria (13). Exclusion criteria were evidence of locally infiltrative or metastatic bladder tumours ( $\mathrm{pT} \geq 2$ ), presence of upper urinary tract tumours, lesions that could not be completely removed transurethrally and presence of other neoplastic, lower urinary tract or major concomitant diseases. Patients who had undergone surgery for benign prostatic obstruction at the same time as surgical treatment for NMIBC were also excluded, along with patients with histologically documented urothelial carcinoma in situ. Only those with a tumour recurrence of the same stage and grade of the initial tumour at diagnosis were enrolled. We enrolled only patients with recurrent urothelial cancer in order to obtain a homogeneous group for analysis. Patients with histologically confirmed pT1G3 were excluded, since management decisions in these patients are critical (15). A recurrent bladder tumour within 3 months of receiving TUR-BT was also a criterion for exclusion. Patients who had previously received intravesical treatment were included, provided this treatment had been administered $>6$ months before TUR-BT. No patient underwent early single intravesical instillation, as suggested by Cai et al (16).
Treatment plan. At least 21 days after TUR-BT, all patients underwent weekly BCG instillations for 6 weeks $\left(5 \times 10^{8}\right.$ colony-forming units in $50 \mathrm{ml}$ saline; OncoTice ${ }^{\circledR}$ BCG, Organon USA Inc.). In accordance with Lamm et al (17), boosters of BCG were given at $3,6,12,18,24,30$ and 36 months. This protocol schedule is the current standard treatment for NMIBC (13). All patients were instructed to retain the drug solution in the bladder for $1 \mathrm{~h}$.

Sample collection and storage. Urine samples were collected from each subject by using a clean catch. A spontaneous morning void urine sample was collected by the patients before TUR-BT and by the controls on enrolment. The urine sample was collected from each subject in order to obtain a basal IL-6 and IL-10 mean value. All samples were stored in aliquots at $-80^{\circ} \mathrm{C}$ until analysis after testing for the presence of inflammatory cells or bacteriuria by using urine dipstick test for nitrites and leukocyte esterase (Multistik Pro ${ }^{\circledR}$ ), as shown by Cai et al (18-19). A positive test result was indicative of urinary tract infection.

Interleukin measurements. At the time of the analysis, urine aliquots were defrosted and centrifuged for $10 \mathrm{~min}$ at $800 \mathrm{rpm}$ and the urinary IL-6 and IL-10 levels were measured in the supernatants. Natural human-produced IL- 6 and IL-10 concentrations were determined in the urine of all patients and controls by solid phase ELISA Quantikine IL-6 Immunoassay and IL-10 Immunoassay, respectively (R\&D Systems, Minneapolis, MN, USA). All samples were measured in duplicate in accordance with the manufacturer's recommendations (www.rndsystems. com/productdetailobjectnameQuantikine.aspx). The median minimal detectable dose of the IL- 6 assay was $0.70 \mathrm{pg} / \mathrm{ml}$ and $3.9 \mathrm{pg} / \mathrm{ml}$ for IL-10. Values were considered normal if under the lowest IL-6 and IL-10 standards, $3.12 \mathrm{pg} / \mathrm{ml}$ and $7.8 \mathrm{pg} / \mathrm{ml}$, respectively. All samples were tested in duplicate by using independent analysis to avoid errors.

IL-6/IL-10. The IL-6 to IL-10 ratio was calculated as a fraction between urinary IL- 6 and IL-10 values, expressed as a number or fraction ranging from 0 to 1 . The cut-off value was determined according to Cai et al (9).

Histopathology. Tumour stage and grade were classified following the 1998 WHO classification and the 2002 TNM classification of malignant tumours, as defined by the International Union Against Cancer. A single genito-urinary pathologist performed the pathological evaluation.

Patient follow-up. Each patient underwent urinary cytology, ultrasonography and cystoscopy every 3 months for the first 12 months following TUR and then every 6 months. Recurrence was determined by papillary formations protruding into the bladder lumen and detected on cystoscopy, or by the presence of neoplastic tissue diagnosed following vesical biopsy. Every recurrence was pathologically confirmed. Time to the first recurrence was defined as the time from randomisation to the date of the first recurrence. Progression was defined as an increase in tumour stage or grade (13).

Statistical analysis. As the null hypothesis, we assumed that there was no difference among the groups in terms of 
recurrence-free time or recurrence rate and that the IL-6/IL-10 had no impact on predicting the response to BCG therapy. The Fisher's exact test and the Chi-square $\chi^{2}$ test were used to assess the significance of all parameters, and $\mathrm{p}<0.05$ was considered to indicate a statistically significant difference. Kaplan-Meier survival curves and the log-rank test were also used to evaluate survival in NMIBC cases. The $95 \%$ confidence intervals (CI) were calculated for the probability of survival for Kaplan-Meier estimates. The Mann-Whitney test was also used to compare different parameter means. Univariate and multivariate relative risk was calculated by using Cox's proportional hazards regression. Statistical analysis was performed using StatPlus for Apple Macintosh (Analyst Soft, 2001-2009).

\section{Results}

Among 196 consecutive patients undergoing TUR-BT, 81 were enrolled, of which 72 were found to be eligible for the present study. This trial ultimately included 72 patients and 49 controls. Patient characteristics, clinical and laboratory data are described in Table I.

Results at enrolment. No patient tested positive in the urine dipstick test. IL-6 and IL-10 were $74.13 \mathrm{pg} / \mathrm{ml}(22.84 \pm$ SD) and $196.28 \mathrm{pg} / \mathrm{ml}(36.59 \pm \mathrm{SD})$, respectively, among the patients, while IL-6 and IL-10 were $3.11 \mathrm{pg} / \mathrm{ml}(1.13 \pm$ SD) and $5.78 \mathrm{pg} / \mathrm{ml}(2.17 \pm \mathrm{SD})$, respectively, in the control group. The IL-6/IL-10 mean value was $0.13(0.13 \pm \mathrm{SD})$ among the patients (Fig. 1). At the baseline, 38 out of $72(52.7 \%)$ patients showed an IL-6/IL-10 of $\leq 0.09$ while 34 (47.3\%) showed an IL-6/IL-10 of $>0.10$. The IL-6/IL-10 ratio was not calculated in the control group. All patients were then split into 2 groups: 38 patients to group A (IL-6/IL-10 $\leq 0.09$ ) and 34 to group B (IL-6/IL-10 >0.10). In group A the IL-6/IL-10 mean value was $0.05(0.01 \pm \mathrm{SD})$ while in group B it was $0.17(0.11 \pm \mathrm{SD})$.

Follow-up results. All patients correctly underwent BCG intravesical therapy. Nineteen patients had mild adverse effects, which did not require treatment suspension and which were cured with antibiotic and anti-inflammatory drugs. At the end of the follow-up period (mean, 53.7 months; ranging from 49 to 58 months), all patients were alive, 39 (54.1\%) without recurrence, while $33(45.9 \%)$ had at least one recurrence. Three patients showed progression in stage and/or grade: 2 pTaG 2 to pT1G3 (time to progression, 8 months) and 1 pTaG1 to pT1G3.

\section{Results according to the groups}

Group A (IL-6/IL-10 $\leq 0.09)$. Among patients with IL-6/ IL-10 $\leq 0.09,23$ out of $38(60.5 \%)$ showed at least one recurrence (mean, 6.2 months; ranging from 3 to 16 months), while $15(39.5 \%)$ experienced no recurrence (mean, 13.1 months; ranging from 11 to 16 months). Among the patients with at least one recurrence at follow-up, 2 patients showed progression in stage and grade ( 2 pTaG2 to pT1G3).

Group $B(I L-6 / I L-10>0.10)$. Among patients with IL-6/ IL-10 $>0.10,10$ out of $34(29.4 \%)$ showed at least one recurrence (mean, 12.3 months; ranging from 10 to 14 months) while $24(70.6 \%)$ had no recurrence (mean, 16.1 months' ranging 11 from to 17 months). Among the patients with at least one recurrence at follow-up, one patient showed grade
Table I. Summary of clinical and pathological patient and control data.

A, Patient and control characteristics

\begin{tabular}{lc} 
No. of patients & 72 \\
No. of controls & 49 \\
Median patient age (range) & $71.3(52-79)$ \\
Median control age (range) & $68.7(57-75)$ \\
Gender (patients) & \\
Male & $70(97.2)$ \\
Female & $2(2.8)$ \\
Gender (controls) & \\
Male & $49(100.0)$ \\
Female & $0(0.0)$ \\
\hline
\end{tabular}

B, Patient anamnestic, pathological and clinical data

Number of recurrences/year

1

$8(11.1)$

2

$\geq 3$

$49(68.0)$

Number of lesions

1

$11(15.2)$

2

$25(34.7)$

$\geq 3$

$36(50.1)$

Diameter of lesion

(if multiple, diameter of the largest)

$<3 \mathrm{~cm}$

$44(61.1)$

$\geq 3 \mathrm{~cm}$

Stage

pTa

pT1

Grade

G1

G2

$26(36.2)$

G3

$38(52.7)$

Previous intravesical therapy

No treatment

$11(15.2)$

BCG

$25(34.7)$

Chemotherapy $^{\mathrm{a}}$

$36(50.1)$

C, Control clinical data

Urological diseases

TUR-P

$49(100)$

Anamnestic and clinical data of all enrolled patients and controls. Data in parentheses are percentages. BCG, bacillus Calmette-Guérin; TUR-P, transurethral resection of the prostate. ${ }^{a}$ Chemotherapy with $30 \mathrm{mg}$ epirubicin.

progression (1 pT1G1 to pT1G3). All follow-up data pertaining to the groups are detailed in Table II. 
Table II. Follow-up results according to IL-6/IL-10 value.

\begin{tabular}{|c|c|c|c|c|c|}
\hline \multirow[b]{2}{*}{ Group } & \multirow[b]{2}{*}{ IL-6/IL-10 } & \multicolumn{3}{|c|}{ Status at follow-up } & \multirow[b]{2}{*}{ Total } \\
\hline & & VNED & $\begin{array}{c}\text { VREC } \\
\text { with progression }\end{array}$ & $\begin{array}{c}\text { VREC } \\
\text { without progression }\end{array}$ & \\
\hline A & $\leq 0.09$ & 15 & 2 & 21 & 38 \\
\hline B & $>0.10$ & 24 & 1 & 9 & 34 \\
\hline Total & & 39 & 3 & 30 & 72 \\
\hline
\end{tabular}

Follow-up results according to IL-6 and IL-10 ratio value. IL-6, interleukin-6; IL-10, interleukin-10. VNED, alive without recurrence; VREC, alive with at least one recurrence.

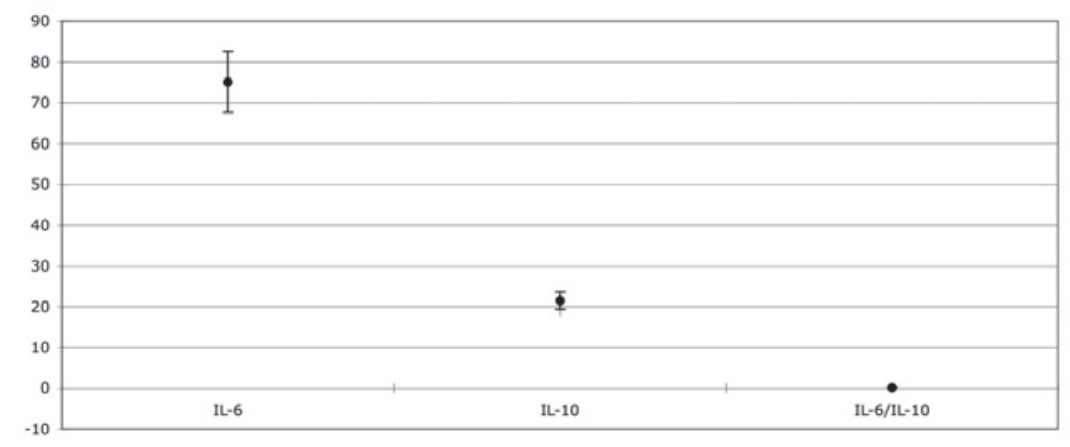

Figure 1. Box-plot showing the mean values of interleukin-6, interleukin-10 as well as interleukin-6 and interleukin-10 ratio among all enrolled patients.

Statistical difference between IL-6/IL-10, other clinical or pathological factors and follow-up results. No correlation between anamnestic or pathological factors and the IL-6/IL-10 value was reported. A statistically significant difference between the IL-6/IL-10 value and the response to BCG therapy (as status at follow-up) was found ( $\mathrm{p}=0.016, \mathrm{df}=1, \chi^{2}=5.800$ ). The Kaplan-Meier analysis demonstrated a significant difference in terms of response to BCG between the 2 groups $[\mathrm{p}=0.003$; recurrence rate $(\mathrm{RR})=3.1$; standard error $(\mathrm{SE})=1.18]$ (Fig. 2).

Univariate and multivariate analysis. No clinical or anamnestic parameter had any significant impact on BCG response, as shown by univariate analysis. At multivariate analysis, the IL-6/IL-10 value ( $\mathrm{p}=0.002)$ [IL-6/IL-10 value; hazard ratio (HR), 4.09; 95\% CI, 2.59-6.28] and number of lesions ( $\mathrm{p}=0.03$ ) (3 lesions; HR, 3.31; 95\% CI, 1.38-3.35) were identified as independent prognostic factors of BCG response probability (Table III).

\section{Discussion}

In his review on the management of BCG failures in NMIBC patients, Witjes stated that the response to BCG therapy cannot be accurately predicted on an individual basis (2). In previous years, however, several molecular markers, such as urinary cytokines (20), p53 and pRb expression (6) and loss of heterozygosis on INF- $\alpha$ locus (9), have been proposed as prognosticators of BCG response, even if their clinical usefulness remains to be proven (2). Cai et al recently demonstrated that

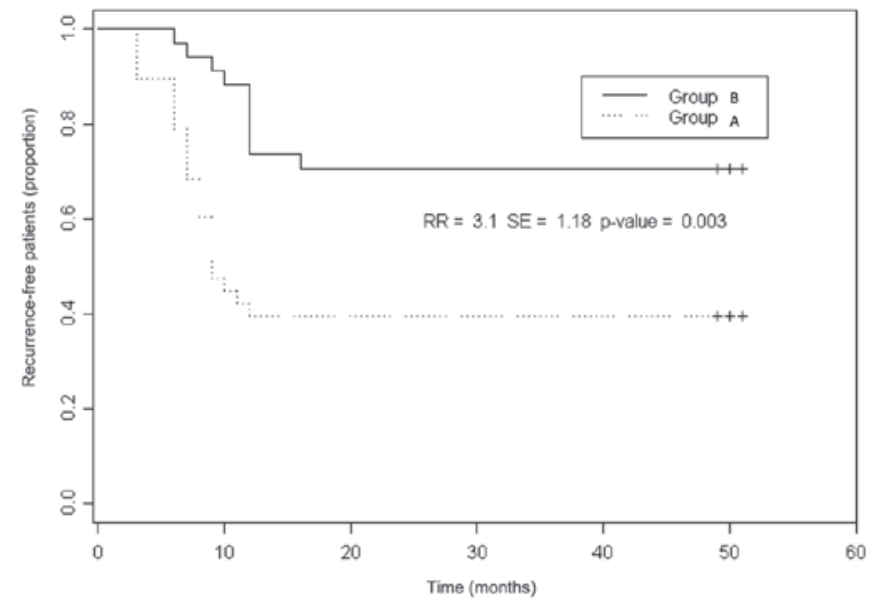

Figure 2. Kaplan-Meier curve analysis performed in order to calculate the probability of being recurrence-free between the 2 groups. RR, relative risk; SE, standard error.

IL-6/IL-10 predicts the risk of recurrence in NMIBC patients (9). In the present study, we have shown that IL-6/IL-10 is an independent prognostic factor of BCG response probability. The rationale of this approach is based on the fact that IL-6 and IL-10 are cytokines overexpressed in bladder carcinoma, and with a role in the interaction between cancer cells and the evironment (21). IL-6 is a multifunctional cytokine produced by cells in response to several inflammatory conditions (22) and has been recognised as a growth factor for the final maturation of $\mathrm{B}$ cells and the differentiation of $\mathrm{T}$ cells (23). 
Table III. Multivariate analysis results of factors affecting recurrence risk in all enrolled patients.

\begin{tabular}{|c|c|c|}
\hline $\begin{array}{l}\text { Categories } \\
\text { (variables) }\end{array}$ & $\begin{array}{l}\text { No. of recurrence-free patients/ } \\
\text { Total no. of patients }(\%)\end{array}$ & $\begin{array}{l}\text { Multivariate analysis } \\
\text { HR }(95 \% \mathrm{CI})(\mathrm{p})\end{array}$ \\
\hline Age & & 0.74 \\
\hline$<65$ years & $23 / 42(54.7)$ & $0.77(0.60-1.43)$ \\
\hline$\geq 65$ years & $16 / 30(53.3)$ & $0.62(0.15-1.16)$ \\
\hline Gender & & 0.71 \\
\hline Male & $38 / 70(54.2)$ & $1.00(0.18-1.55)$ \\
\hline Female & $1 / 2(50.0)$ & $1.21(0.75-1.16)$ \\
\hline Number of recurrences/year & & 0.25 \\
\hline 1 & $5 / 8(62.5)$ & $0.99(0.12-1.67)$ \\
\hline 2 & $6 / 15(40.0)$ & $0.74(0.10-1.19)$ \\
\hline$\geq 3$ & $28 / 49(57.1)$ & $1.15(0.47-2.00)$ \\
\hline Diameter of lesions & & 0.89 \\
\hline$<3 \mathrm{~cm}$ & 29/44 (65.9) & $1.01(0.15-1.23)$ \\
\hline$\geq 3 \mathrm{~cm}$ & $10 / 28(35.7)$ & $2.21(2.10-3.02)$ \\
\hline Grade & & 0.09 \\
\hline G1 & $3 / 8(37.5)$ & $0.97(0.12-1.13)$ \\
\hline G2 & $16 / 26(61.5)$ & $1.10(0.23-1.24)$ \\
\hline G3 & $20 / 38(52.6)$ & $1.99(0.78-2.32)$ \\
\hline Number of lesions & & 0.03 \\
\hline 1 & 10/11 (90.9) & $0.62(0.24-1.08)$ \\
\hline 2 & $13 / 25(52.0)$ & $1.29(0.61-1.99)$ \\
\hline$\geq 3$ & $16 / 36(44.4)$ & $4.22(2.62-3.95)$ \\
\hline IL-6 levels & & 0.62 \\
\hline Normal $(\leq 3.12$ pg/ml $)$ & 21/37 (56.7) & $1.68(1.50-2.87)$ \\
\hline Abnormal & 18/35 (51.4) & $1.12(0.20-2.12)$ \\
\hline IL-10 levels & & 0.71 \\
\hline Normal $(\leq 7.8 \mathrm{pg} / \mathrm{ml})$ & 16/21 (76.1) & $0.87(0.74-1.09)$ \\
\hline Abnormal & 23/51 (45.0) & $1.09(0.19-1.20)$ \\
\hline IL-6/IL-10 & & 0.002 \\
\hline$\leq 0.09$ & 15/38 (39.4) & 0.65 (0.29-1.19) \\
\hline$>0.10$ & $24 / 34(70.5)$ & $3.87(2.81-4.98)$ \\
\hline
\end{tabular}

Multivariate analysis results of factors affecting recurrence risk in all enrolled patients. Data in parentheses are percentages. HR, hazard ratio, 95\% CI, 95\% confidence interval. IL-6, interleukin-6; IL-10, interleukin-10.

IL-6 is crucial in supporting systemic host response to tissue injury (24). Mulé et al described the anti-tumour effect of IL-6 on lung cancer in mice, confirming that the induction of host immunological response is central in the reaction to cancer (25). Moreover, several studies have demonstrated that IL-10 has the physiological role of downregulating cell-mediated immunity, resulting in the improvement of tumour immune escape (26). Nadler et al reported that IL-10 is an important modulator of immune-mediated events in vivo and suggested that efforts to downmodulate this inhibitory cytokine may be of therapeutic value (27). The theory that IL-6/IL-10 is a good prognostic marker for use in clinical practice was based on the following reasons: i) high levels of IL-10, with consequent decrease in IL-6/IL-10, indicate tumour immunosuppression, eliminating the ability of immunocompetent cells to respond to the tumour (26); ii) IL-10 is known to be a promoter of immune dysregulation, with the enhancement of Th2 cells (27). Indeed, patients with bladder cancer appear to develop a Th2 dominant status with a deficient-type immune response due to an increase in IL-10 levels (27). This immune dysregulation should be proved by altered IL-6/IL-10; iii) the decrease in IL-6 levels and increase in IL-10 levels are evidence of an altered host immune response to the tumour, confirmed by cell activation due to BCG (26). The release of immunosuppressive IL-10 during BCG therapy reduces inflammation and anticancer response (28). In addition, IL-6 and IL-10 are promoters of two different immune response pathways, i.e. Th1 and Th2, and allow the evaluation of host immunological response to the tumour $(26,29)$. The present study, even though conducive for a better understanding of the correlation 
between urothelial bladder cancer cells and the host immune system, had a number of limitations that should be taken into account. First of all, the number of the patients enrolled was small, even if a statistical significance was obtained. Secondly, a group of patients undergoing alternative intravesical therapies, i.e. mitomycin or epirubicin, was lacking. Future studies are mandatory to confirm the present findings.

The current study highlights the role of IL-6/IL-10 in predicting the response to BCG therapy in NMIBC patients. We put forward the feasibility and usefulness of IL-6/IL-10 as a clinical marker, in association with traditional clinicopathological factors, in planning a more appropriate follow-up schedule for NMIBC patients.

\section{Acknowledgements}

Special thanks to Professor John Denton for manuscript language revision and to all members of the Santa Maria Annunziata Hospital STD Senter, for their excellent laboratory assistance.

\section{References}

1. Sylvester RJ, van der Meijden AP, Oosterlinck W, Witjes JA, Bouffioux C, Denis L, Newling DW and Kurth K: Predicting recurrence and progression in individual patients with stage Ta T1 bladder cancer using EORTC risk tables: a combined analysis of 2596 patients from seven EORTC trials. Eur Urol 49: 466-465, 2006.

2. Witjes JA: Management of BCG failures in superficial bladder cancer: a review. Eur Urol 49: 790-797, 2006.

3. Punnen SP, Chin JL and Jewett MA: Management of bacillus Calmette-Guerin (BCG) refractory superficial bladder cancer: results with intravesical BCG and Interferon combination therapy. Can J Urol 10: 1790-1795, 2003.

4. Kim JC and Steinberg GD: Medical management of patients with refractory carcinoma in situ of the bladder. Drugs Aging 18 335-344, 2001

5. Herr HW, Wartinger DD, Fair WR and Oettgen HF: Bacillus Calmette-Guerin therapy for superficial bladder cancer: a 10-year followup. J Urol 147: 1020-1023, 1992.

6. Esuvaranathan K, Chiong E, Thamboo TP, Chan YH, Kamaraj R, Mahendran R and Teh M: Predictive value of p53 and pRb expression in superficial bladder cancer patients treated with BCG and interferon-alpha. Cancer 109: 1097-1105, 2007.

7. Huguet J, Crego M, Sabate S, Salvador J, Palou J and Villavicencio H: Cystectomy in patients with high risk superficial bladder tumours who fail intravesical BCG therapy: pre-cystectomy prostate involvement as a prognostic factor. Eur Urol 48: 53-59, 2005.

8. Ahirwar D, Kesarwani P, Manchanda PK, Mandhani A and Mittal RD: Anti- and proinflammatory cytokine gene polymorphism and genetic predisposition: association with smoking, tumor stage and grade, and bacillus Calmette-Guérin immunotherapy in bladder cancer. Cancer Genet Cytogenet 184: 1-8, 2008

9. Cai T, Nesi G, Dal Canto M, Tinacci G, Mondaini N, Piazzini M, Geppetti P and Bartoletti R: Loss of heterozygosis on IFN-alpha locus is a prognostic indicator of bacillus Calmette-Guerin response for nonmuscle invasive bladder cancer. J Urol 183: 1738-1743, 2010.

10. Zhang Y, Mahendran R, Yap LL, Esuvaranathan K and Khoo HE: The signalling pathway for BCG-induced interleukin- 6 production in human bladder cancer cells. Biochem Pharmacol 63: 273-282, 2002.

11. Luo Y, Han R, Evanoff DP and Chen X: Interleukin-10 inhibits Mycobacterium bovis bacillus Calmette-Guérin (BCG)-induced macrophage cytotoxicity against bladder cancer cells. Clin Exp Immunol 160: 359-368, 2010
12. Chen FH, Crist SA, Zhang GJ, Iwamoto $Y$ and See WA: Interleukin- 6 production by human bladder tumor cell lines is up-regulated by bacillus Calmette-Guérin through nuclear factorkappaB and Ap-1 via an immediate early pathway. J Urol 168: 786-97, 2002

13. Oosterlinck W, Lobel B, Jakse G, Malmström P, Stöckle M and Sternberg C; European Association of Urology (EAU) Working Group on Oncological Urology: Guidelines on bladder cancer. Eur Urol 41: 105-112, 2002.

14. Herr HW and Dalbagni G: Defining bacillus Calmette-Guerin refractory superficial bladder tumors. J Urol 169: 1706-1708, 2003.

15. Thalmann GN, Markwalder R, Shahin O, Burkhard FC, Hochreiter WW and Studer UE: Primary T1G3 bladder cancer: organ preserving approach or immediate cystectomy? J Urol 172: 70-75, 2004.

16. Cai T, Nesi G, Tinacci G, Zini E, Mondaini N, Boddi V, Mazzoli S and Bartoletti R: Can early single dose instillation of epirubicin improve bacillus Calmette-Guerin efficacy in patients with nonmuscle invasive high risk bladder cancer? Results from a prospective, randomized, double-blind controlled study. J Urol 180: 110-115, 2008.

17. Lamm DL, Crawford ED, Blumenstein B, Crismann JD, Montie JE, Gottesman JE, Lowe BA, Sarosdy MF, Bohl RD, Grossman HB, Beck TM, Leimers JT and Crawford ED: Maintenance BCG immunotherapy for recurrent Ta, T1 and Tis transitional cell carcinoma of the bladder: a randomized prospective Southwest Oncology Group study. J Urol 163: 1124-1129, 2000.

18. Cai T, Mazzoli S, Meacci F, Nesi G, Geppetti P, Malossini G and Bartoletti R: Human papillomavirus and non-muscle invasive urothelial bladder cancer: Potential relationship from a pilot study. Oncol Rep 25: 485-489, 2011.

19. Cai T, Piazzini M, Nesi G, Taddei I, Sardi I, Detti B, Mondaini N, Dal Canto $\mathrm{M}$ and Bartoletti R: E-cadherin mRNA expression analysis in evaluating the natural history of urothelial bladder cell carcinoma: Results from a long-term follow-up study. Oncol Rep 17: 925-930, 2007.

20. Thalmann GN, Sermier A, Rentsch C, Mohrle K, Cecchini MG and Studer UE: Urinary interleukin- 8 and 18 predict the response of superficial bladder cancer to intravesical therapy with bacillus Calmette-Guerin. J Urol 164: 2129-2133, 2000.

21. Watanabe E, Matsuyama H, Matsuda K, Ohmi C, Tei Y, Yoshihiro S, Ohmoto Y and Naito K: Urinary interleukin-2 may predict clinical outcome of intravesical bacillus Calmette-Guerin immunotherapy for carcinoma in situ of the bladder. Cancer Immunol Immunother 52: 481-486, 2003.

22. Barton BE: Interleukin-6 and new strategies for the treatment of cancer, hyperproliferative diseases and paraneoplastic syndromes. Expert Opin Ther Targets 9: 737-752, 2005.

23. Liang B, Gardner DB, Griswold DE, Bugelski PJ and Song X: Anti-interleukin-6 monoclonal antibody inhibits autoimmune responses in a murine model of systemic lupus erythematosus. Immunology 119: 296-305, 2006.

24. Seguchi T, Yokokawa K, Sugao H, Nakano E, Sonoda T and Okuyama A: Interleukin- 6 activity in urine and serum in patients with bladder carcinoma. J Urol 148: 791-794, 1992.

25. Mulé JJ, McIntosh JK, Jablons DM and Rosenberg SA: Antitumor activity of recombinant interleukin 6 in mice. J Exp Med 171: 629-636, 1990.

26. Sabat $\mathrm{R}$ and Asadullah $\mathrm{K}$ : Interleukin-10 in cancer immunity. In: Cancer Immune Therapy: Experiences and Future Strategies. Stuhler G and Walden P (eds). Wiley-VCH Verlag GmbH, Weinheim, Germany, pp223-231, 2002.

27. Nadler R, Luo Y, Zhao W, Ritchey JK, Austin JC, Cohen MB, O'Donnell MA and Ratliff TL: Interleukin 10 induced augmentation of delayed-type hypersensitivity (DTH) enhances Mycobacterium bovis bacillus Calmette-Guérin (BCG) mediated antitumour activity. Clin Exp Immunol 131: 206-216, 2003.

28. Agarwal A, Verma S, Burra U, Murthy NS, Mohanty NK and Saxena S: Flow cytometric analysis of Th1 and Th2 cytokines in PBMCs as a parameter of immunological dysfunction in patients of superficial transitional cell carcinoma of bladder. Cancer Immunol Immunother 55: 734-743, 2006.

29. Suttmann H, Jacobsen M, Reiss K, Jocham D, Bohle A and Brandau S: Mechanisms of bacillus Calmette-Guerin mediated natural killer cell activation. J Urol 172: 1490-1495, 2004. 